\title{
A CONCEPT OF IN-PROCESS MEASUREMENT SYSTEM FOR SPLINE FORMING
}

\author{
Michal Wieczorowski ${ }^{1}$, Tim Eichner $^{2}$, Ingo Lindner ${ }^{2}$, Alejandro Pereira ${ }^{3}$ \\ 1 Poznan University of Technology, Poznan, Poland \\ ${ }^{2}$ Hexagon Metrology, Wetzlar, Germany \\ 3 University of Vigo, Vigo, Spain \\ Corresponding author: \\ Michal Wieczorowski \\ Poznan University of Technology \\ Division of Metrology and Measurement Systems \\ Piotrowo 3, 60-965 Poznan, Poland \\ phone: $(+48) 61$ 665-35-70 \\ e-mail:michal.wieczorowski@put.poznan.pl
}

Received: 29 April 2015

Accepted: 8 May 2015

\begin{abstract}
In the paper a possibility of in-process inspection of gear and spline shafts during forming was discussed. Different methods of forming toothed elements were discussed as well as the other technologies were mentioned. Coordinate measurement technique as a way of post process inspection was presented. However, it is not possible to use it for checking features during forming process. To do it different sensors were used and their combinations were described.
\end{abstract}

KEYWORDS

gear, spline, forming, coordinate metrology, in-process inspection.

\section{Introduction}

Modern production technology for the manufacture of splined components is complex. For this purpose all currently known production technologies are used. The production of toothed parts is in general distinguishable into the manufacturing of individual parts and mass production. A single component or a small amount of splined components is manufactured usually by machining. In this case various metal cutting methods are used. In the context of series production large numbers are usually produced in a combination of different technologies. Here at the beginning of the production chain it is primary forming or metal forming. This means that, for example, through the primary forming or metal forming a preform is produced.

The final shape is then formed by subsequent metal cutting or other metal forming processes [1]. The structure of such production lines, depends on the geometry of the toothed part, the material from which the part is made, and the quality requirements in relations to the final geometry. Each series produc- tion is dominated by questions of production efficiency. In this sense, individual parts or small quantities of toothed components cannot be made through a preform by primary forming or metal forming. The arising high tool costs would negatively affect economics of such production. For individual parts in this sense the production line starts with, for example, semi-finished bars. Figure 1 shows a compilation of various technologies and processes that are used in modern gear manufacturing. Starting from a preform which has been produced e.g. by molding or forming, it is made by rotating the output body. Then the creation of teeth is realized by machining or forming. Onto the mention of various intermediate steps, in particular the metrological examination of various production steps has been abdicated.

Splines are mass parts that are required, for example in the automotive industry in very large numbers. Spline shafts are usually paired with an internal gear hub. This component combination is called shaft-hub connection (Fig. 2) and are used for the transmission of torque allowing axial displacement of the shaft against the hub. 


\section{METHOD for manufacturing toothed machine elements}

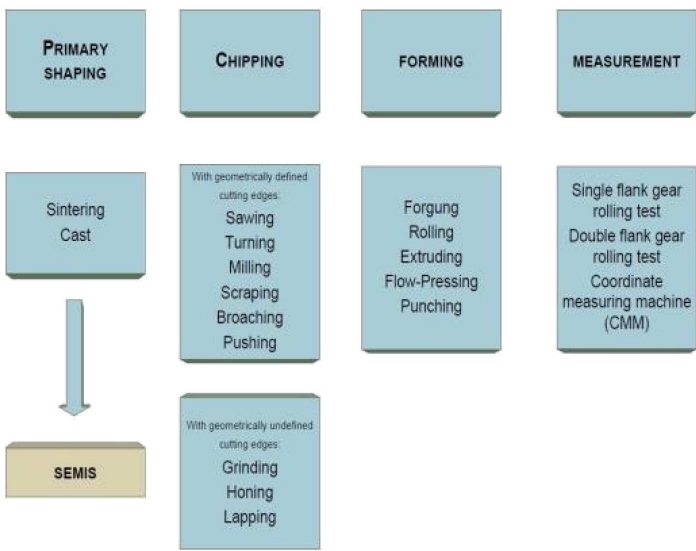

Fig. 1. Compilation of various technologies and processes for manufacturing of gears.

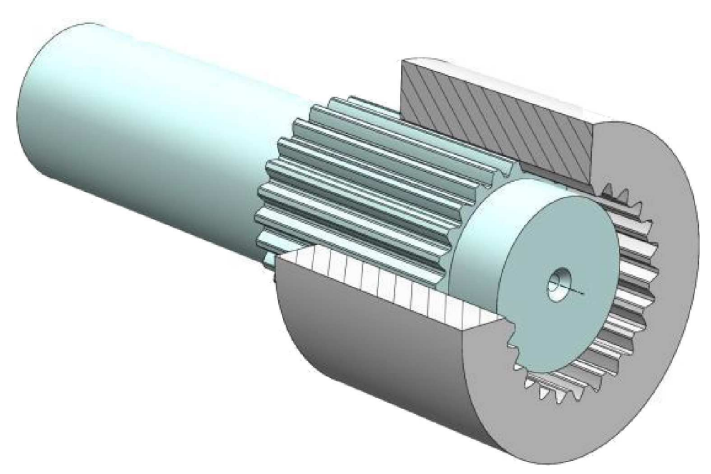

Fig. 2. Shaft-hub connection.

The aspired geometric ideal of this pairing is that the center of the externally toothed shaft and the center of the internal gear hub in the assembled state are identical.

Deviation from the ideal pairing of hub and shaft would have in technical use significant negative consequences. Symmetry of the load distribution on both machine elements would be disturbed. Geometric points of contact or contact surfaces of both components should therefore be absolutely symmetrically regarding an identical center after fabrication. This means technically that the functional elements (teeth) on the two components based on the same center in shape and position should be made absolutely identical. Only then the main functional surfaces of both teeth on the same geometric locations in the space have the ideal mechanical contact. This is the production - technical challenge that must be overcome in the mass production of such gear pairings $[2]$.

\section{Metal forming for manufacturing of gears and splines}

Metal forming is a very old technology for shaping components. One of the oldest methods of forming is forging. Thereby, through one or more tools, required energy for shaping is introduced into material. At all methods of forming mechanical energy changes the shape of a material. The volume of the processed material remains constant, regardless of whether it is bulk or sheet-shaped material [3]. Toothed machine elements are mostly made of bulk material. A distinction is made into hot, warm and cold forging. During hot or semi-hot forming, the volume is deformed dependent on the material at temperatures of approximately $600-1200^{\circ} \mathrm{C}$, while cold forming takes place at room temperature. Nowadays, toothed machine elements are produced chipless through forging, extruding or rolling. The desired geometrical quality of the components determines the employed manufacturing process. The advantages of all forming processes are i.e. material savings and associated solidification of the material structure through metal forming. Forging is used primarily for the production of a preform. The resulting geometric components quality does not always match the required quality standard. The required quality is usually achieved by subsequent cutting processes. Extrusion is used mostly for cylindrical components with a long axial meshing. And rolling processes are always used wherever rotationally symmetric components are to be toothed. Often, the reshaping is followed by a carburizing and/or a hardening process, so that the surface layer of the teeth have better abrasion behavior. For the highest geometric requirements the reshaping can also be followed by a cutting process such as grinding.

A clear statement which method or combination of methods is used for specific types of gear components cannot be made. The variety of different geometric shapes of such components and the desired quality characteristics are too different. The goal of any production must be to work with the lowest expenses. Consequently, the technical necessary production steps are also evaluated in terms of costs. As long as, the required technical function of the toothed component is ensured, different combinations of manufacturing steps and used methods can result in a success. The question of the success of a production is closely linked to the issue of quality control of production. Mass production and technical standards for the quality of manufactured components include various trade-offs. Mass production vs. part accuracy and part accuracy vs. material properties are particularly important for cutting produc- 
tion, the key challenge. Besides many other factors the metal forming is determined through the material properties of the deformed material and the unavoidable tool abrasion [4]. Unwanted variations of the composition of the formed material or changes of the tool shape thru abrasion can significantly affect the resulting quality. This statement concerns the geometry of the component after forming, as well as the then existing mechanical component properties. At the forming production of toothed machine elements, this relationship is of particular importance. The proper function of teeth is directly dependent on the geometric quality of the teeth. By which the large need for control of forming production is explained. Mass production and quality control are another conflict, which takes a special importance while manufacturing toothed machine elements. Short production times that are required in connection with mass production, and great loss of time through quality control is a contradiction in terms. Through this, the economics of production is significantly influenced ultimately. The large amount of time which has to be provided for the correct geometric control of a toothed element, allows just only random checks after the production of a component series. Therefore, the post-process control can only record individual components. Consequently, a comprehensive inspection of all parts is only possible within the manufacturing process. The challenge for the future is to control the mass production of toothed machine elements completely by monitoring the forming process [5]. For our own research test samples were manufactured under controlled conditions. The sample material came from a batch of steel production and was examined metallurgical to its composition. Then the used sequence of manufacturing steps begins for example with rod-shaped carbon steel C15. Accordingly long pieces of this material were annealed ( $\mathrm{HV}$ $\sim 130$ ) and shaped to predetermined dimensions by turning. The desired geometry of the blank is determined by the method which is used for the production of the toothed component. In this research, it was the rolling according to the WPM method. Here the teeth are formed without cutting through two internally toothed tools. The roll samples were rolled in the cold state (room temperature).

\section{Rolling process}

Rolling process includes the area of the pressure forming processes. In these methods, mainly compressive stresses resulting in the forming zone [6]. The rolling processes used for manufacturing toothed components are usually divided by the geometric shape of the applied tools. The methods are differed into the ones with rotationally symmetrical external toothed tools (PeeWee), with flat back shaped tools (RotoFlow), with internal toothed tools (WPM) and the so called planetary rolling method (Grob). The two oldest methods, PeeWee and RotoFlow were developed for non-cutting thread production end of the 1920-ies. A typical component is the screw, as it is produced worldwide for many decades. The screw is a cylindrical toothed machine element, which includes i.e. an essential geometric functional element, the thread. The technological relationships and sequence of manufacturing steps are very similar to the sequence in modern manufacturing of toothed components. Starting from a cylindrical preform, the blank, portions of the preform are formed to the desired functional elements, threads or gears. The screw is a typical mass part; the production is based almost exclusively on the metal forming. For screw production about 20 years ago different process monitoring systems have been developed. Without the monitoring a modern screw production is hard to imagine today. However, the qualitative requirements for the process monitoring of a screw production are significantly lower than in the manufacturing of toothed machine elements. The process monitoring of screw production is based solely on the detection of technical machine data during the forming. At all the aforementioned rolling processes enters a wedge-shaped tool in the surface contour of the blank and displaces the material.

Rolling according WPM method is based on two internally toothed rolling tools which move on symmetric eccentric courses. The blank is held between tips and turns driven by the machine. The rotating blank is fed between the rolling tools (Fig. 3). The tool teeth grasp the material, make the blank rotate and forms teeth on the entire circumference of the workpiece. The resulting teeth on the blank per feed step is formed completely. After every stroke the tools open and release the workpiece for the next feed step. The magnitude of the axial extension of the developing serration per stroke is dependent upon the feed rate at which the blank is inserted between the tools.

The rolling according to the WPM method of prof. Z. Marciniak is characterized by two internally toothed rolling tools [7]. The tools move in symmetrical and circular eccentrically courses, as shown in the Fig. 4.

The rolling process starts with rotating rolling tools. In accordance with the machine set up of the eccentricity, the tools teeth approach (2) and remove at every stroke (7) from the center of a cylindrical 
blank. The blank rotates with the same peripheral speed as the tools and is inserted axially between the rolling tools. The tool teeth engage the blank and penetrate into the material $(2,3,4,5,6,7)$, so that the tool teeth get reproduced in the material. At each tool stroke arise therefore a rolling phase and a phase in which the tools are opened $(1,8)$. In the open state of the tools the blank is axially advanced and the rolling process begins again. At each tool stroke the teeth penetrate into the material to their possible maximum depth so that the entire circumference of the roll sample a complete set of teeth is formed. The axial extent of the resulting spline shaft is given by the number of tool strokes per time and the machine side set up of the feed rate with which the workpiece is advanced axially.

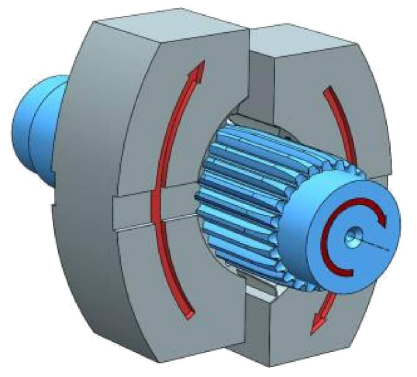

Fig. 3. A scheme of a WPM method.
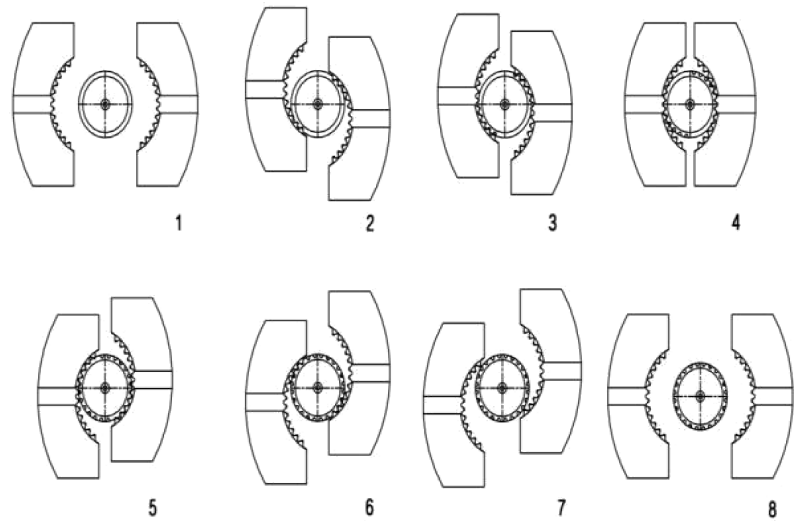

Fig. 4. WPM: motion principle tools/workpiece.

The above briefly described rolling methods cover the entire area of the geometric possibilities to use just a solid tool contour and only a linear or rotary motion to stress a cylindrical blank surface so that a set of teeth is formed by forming. Industrial practice in dealing with these rolling process shows that not each of these methods is suitable for every type of toothed components [8]. Assuming appropriate performance of the machine technology and stability of the rolling tools arise nevertheless significant differences. The size of the teeth and producible quality of the achievable teeth are set process specific limits. Responsible for this are the resulting forces of the deformation. These are dependent on the strength of the metallic material and the contact surfaces between the tool and the workpiece material during forming. The reaction forces as a result of metal forming act on the workpiece. The workpiece attempts to evade these forces and thereby loses its ideal location between the tools. Whereby, a loss in quality of the resulting gear quality must be accompanied. In this respect, the guiding characteristics of the tools referring to the stressed workpiece are of dominant importance. Comparing the three methods described above in this regard, it should be noted that the externally toothed tools (PeeWee) overlap the workpiece teeth the least, the rack shaped tools (RotoFlow) a little more and the internally toothed tool (WPM) the largest. The internal gear tools of the WPM process guide the work piece during the forming the best. This is one of the reasons that, with this method, spline shafts on the order module $\geq 2.5$ can be rolled, which is with the two other methods not possible.

\section{Coordinate measuring technology}

The principle of coordinate measuring technique is based on the fundamental relationship that geometry of each workpiece realized by manufacturing technology, can be described by points in space (Fig. 5). Each point in space is described by three coordinates [9]. These are the geometric lengths (distances) of the point in space relative to the origin of the corresponding coordinate axes. The principle of tac-

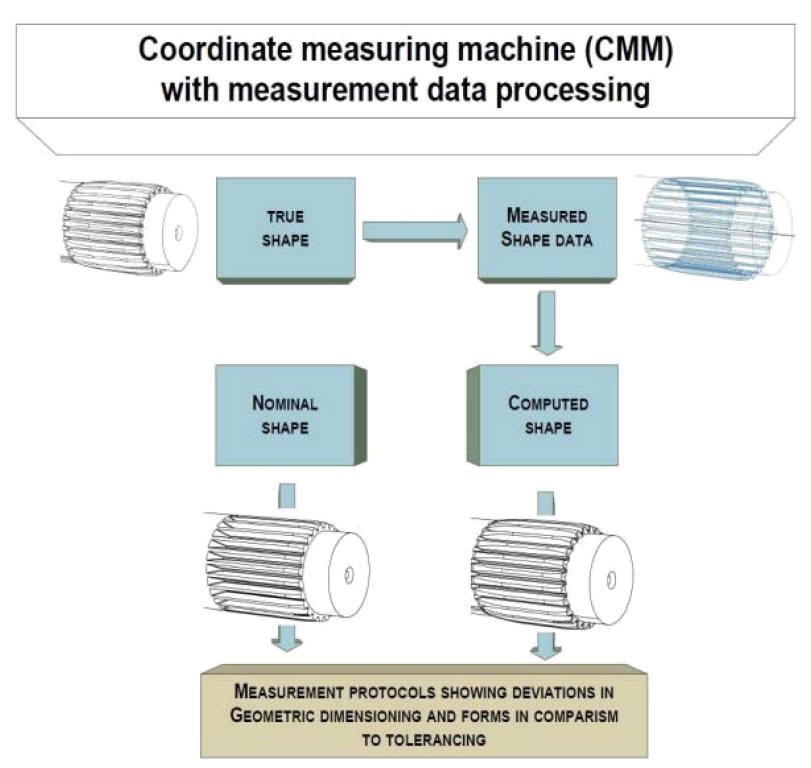

Fig. 5. An idea of coordinate metrology. 
tile three-coordinate measuring technique (Fig. 6) contains the probing of the spatial points by tactile pins of a sensing head. The sensing head realizes the probing and the acquisition of values. A connected computer system takes over and stores the measured values of the probed points in space, links them to geometric elements, determines the actual geometry, conducts a target/actual performance comparison if necessary and calculates/rates and documents the resulting deviations.

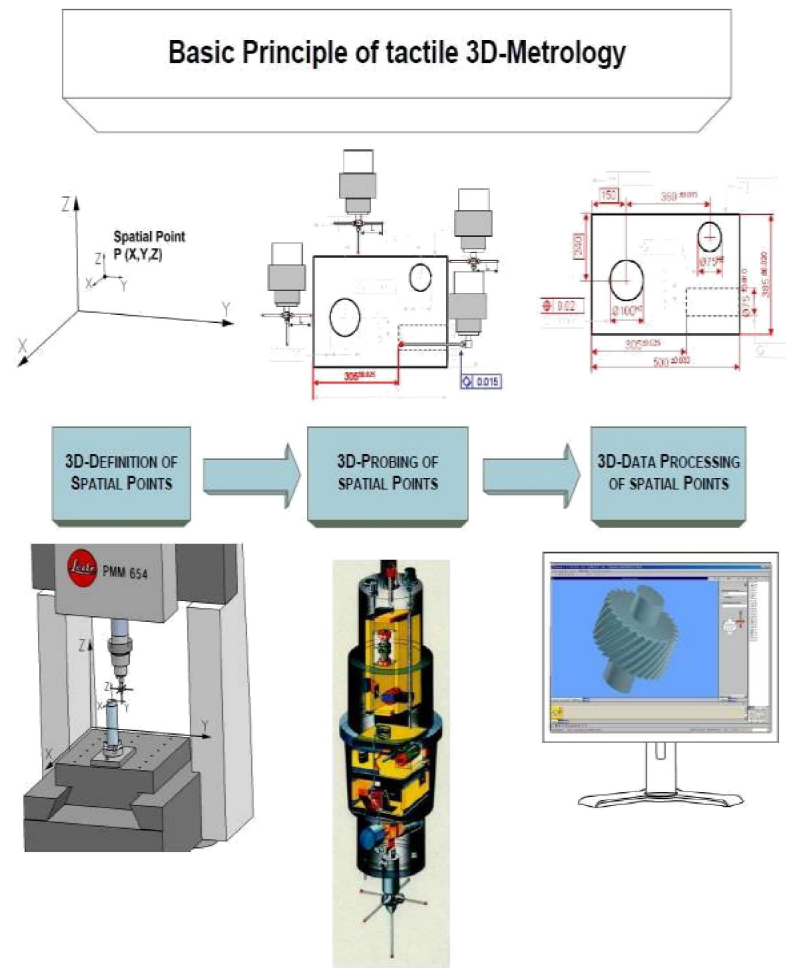

Fig. 6. A principle of tactile 3-D coordinate metrology.

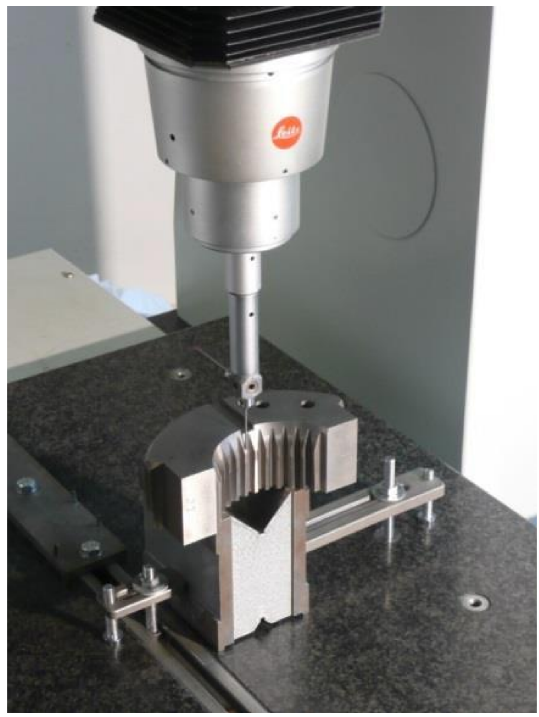

Fig. 7. An idea of coordinate metrology.
Coordinate metrology performed on Coordinate Measuring Machines (CMM) is the most common way of inspecting gears $[10,11]$. It gives a lot of information in relatively short time, though definitely not short enough to make $100 \%$ control. The results obtained by means of CMMs are accurate enough to be sure whether the toothed element is ok or not. Still, this kind of measurement is made after machining and it is very difficult to use it as a control tool in mass production. Figure 7 shows a forming tool for spline shafts being measured on a Coordinate Measuring Machine.

\section{A concept of in-process acquisition of geometric features}

Previously, post-process geometry acquisition and valuation was described. Hence, a validation of the formed work pieces from a quality point of view is possible. The measurements can also show specific geometrical features which result from the forming process. These are not covered by the form deviations according to standards and yet they are of importance for the final technical functionality of the toothed components formed by the WPM process. Their change in dependence of feed velocity, for example, can be used to describe the forming process. Insofar the acquisition of these geometrical features called peculiarities in the machine could lead to early insights.

The advantage of such an action would enable an early assessment of manufacturing process. A subsequent just-in-time reaction would allow for significant cost reductions [12]. On the base of the geometrical peculiarities detected by face profile measurement described above, it will be considered in the following part, which technical requirements have to be imposed on instrumentation and sensors in order to allow for future in-process monitoring of the WPM metal forming process [13].

Certain typical deviations of shape, position and orientation could be detected in-process online and possibly even corrected in real-time by application of appropriate sensors/devices. Among the geometrical deviations which influence the result of the WPM forming process mainly, the predominant parameters are the following:

- eccentricity,

- pitch,

- tooth trace,

- tooth depth, profile form and tip form.

The possibilities to acquire appropriate measuring quantities, which are able to describe these geometrical deviations and which will allow to per- 
form real time calculations of process quantities and are finally able to predict the further process. The properties of the WPM forming process prohibit the employment of tactile sensors, as the forming process is discontinuous, but yet the workpiece is subject to a continuous rotation. In principle, the use of tactile sensors coupled by means of friction could possibly be considered, but usually these devices feature higher measuring inaccuracy. Only for the purpose of additional tests before or after the process, a tactile sensor could be brought in contact with the workpiece in a fixed measuring position; yet this would have the disadvantage of a complete change of the process and the a redesign of the power train and the feed drive train, in addition to an expansion and redesign of the axis control. Obviously, the recommended means for continuous acquisition of the surface geometry are non-tactile, non-contact distance sensors, which are able to capture the radial distance between their mounting position and the surface of the workpiece, as the primary measuring quantity [14-16]. Some of them can be adapted from surface measurement implementing appropriate scale factor $[17,18]$.

Measuring distance between sensor and surface effect in measurement result, from which the main quantities of the tooth profile geometry can be calculated, which is root diameter and tip diameter on one hand, and some properties of tooth form on the other. By means of additional distance sensors which are to be mounted in a fixed offset from the first sensor, further information regarding tooth trace angle can be derived. A further measuring quantity which is necessary to be acquired, is the current value of the rotational angle of the workpiece. This quantity must be captured to enable an exact mapping of profile distance data to the appropriate location on the circumference of the workpiece surface. Besides the necessity of a distance sensor and a sensor for the rotational angle, the axial position of the workpiece during the forming process is required [19]. For this purpose, the measurement of the feed path versus time would be advantageous, as described in the previous chapters. This allows for tracking the progress of the forming process in axial direction, and, moreover, will allow for an exact mapping of the location of the measuring spot of the distance senor. By means of this third measuring quantity, together with the first two, the geometrical properties Pitch, Tooth height and Tooth face thickness can be determined. Metrological, by choosing these three quantities, a workpiece co-ordinate system is created, which consists of two translator axes and one rotary axis. The configuration of the three axes is shown in Fig. 8. The three workpiece-related axes are designated $\mathrm{R}$, $\mathrm{Z}$ and $\varphi$ in a polar coordinate system.

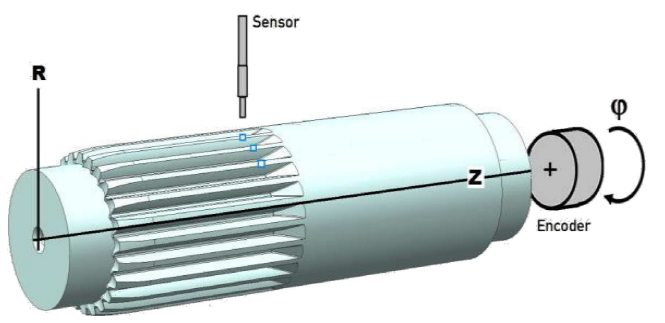

Fig. 8. Sensors in a workpiece coordinate system R, Z, $\varphi$.

For the non-tactile measurement with directcontact sensors, various sensors/instrumentation systems are available [20,21]. Typical examples are laser scanners, laser triangulation systems, laser interferometers, stripe projectors, microwaves, radar or Eddy current. The accuracy and sample rate achievable in a certain application depend on individual properties of the workpiece surface, measurement angle, light and other issues of the particular case of application.

For acquisition of tooth profile, a non-tactile distance sensor is required, which has to be mounted at a most rigid location in the machine frame. To inspect axial position of the workpiece a use of a tactile digital linear position measuring system is possible. CNC machines usually provide such measuring systems for the control of their feed axes, so no additional sensor is necessary. The actual position of the feed axis can be input over standard digital I/O interfaces from the CNC. In the case of the existing WPM-120 Forming Machine, a direct measurement of the workpiece position seemed not to be necessary, as the work-piece is held axially between two tips. So the feed position was measured symmetrically at the feed drive, using two high-accuracy incremental linear measuring systems, which are based on a magnetic $0.5 \mu \mathrm{m}$ scale.

Acquisition of angular position can be made by direct or indirect measurement. For the first, ideally, any non-tactile system of the rotary angle position mounted directly at the workpiece would be sufficient. As the surface and the edges of the workpiece are subject to inconsistencies and variations, measurement would only be possible with a reduced accuracy. There, certain solutions exist, which apply a flexible magnetic scale around the circumference of the workpiece, but then only a non-continuous measurement would be possible, which reduces the resolution considerably. Therefore, the only way remaining to achieve both, a high resolution and a high accuracy, is to measure indirectly at the rotary feed 
drive. Taking into account the particular conditions in the machine, this has the disadvantage of having no rigid coupling of the workpiece to the feed drive, which means that the workpiece - being mounted between tips - may slip-rotate under certain conditions during the forming process cycle. On order to compensate for this disadvantage, additional marks around the circumference are used to sample the rotation of the workpiece every $15^{\circ}$, so it is possible to correct angular slips and to resynchronize the angular position measured at the feed drive. There are various rotatory sensor system available, which are able to measure the angular position with high accuracy. Especially the systems used in CNC machines, measuring absolute or incremental angles, are well known. The practical conditions of the existing feed drive train only allow for measuring the workpiece rotary angle indirectly at the feed drive.

The WPM work cycle can be divided in two phases, the forming phase (Fig. 9 top) and the feed phase (Fig. 9 bottom). During the feed phase, both the workpiece rotational drive and the axial feed drive are active at the same time; at the beginning of the forming phase, both tools get in contact with the workpiece and will block the axial feed against an overload function clutch.

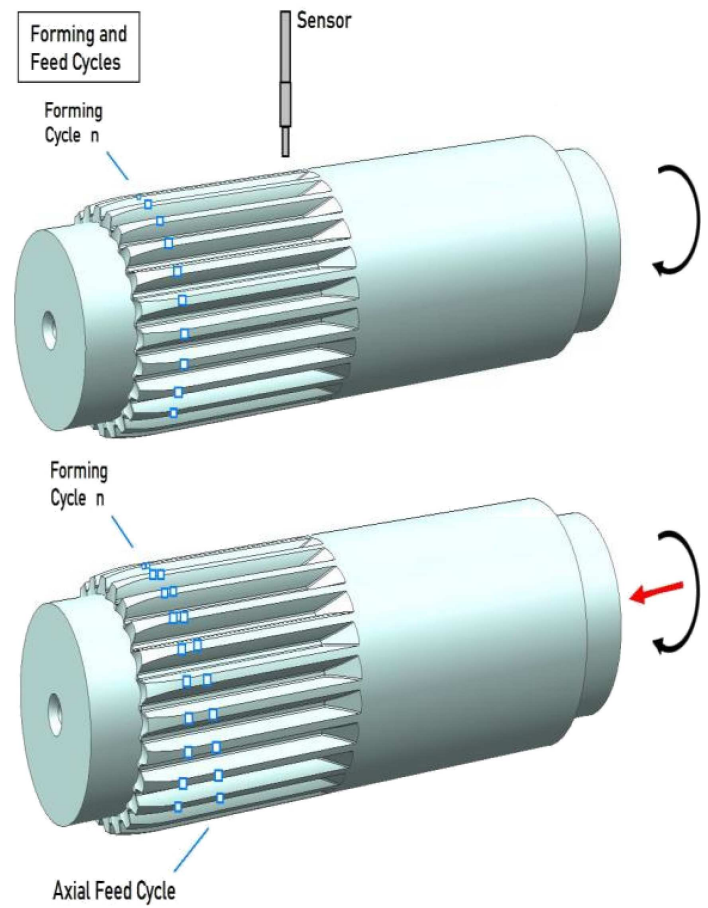

Fig. 9. Sampling sequence in: forming phase $\mathrm{n}$ (top), axial feed phase (bottom).

The sensor signals measured vs. time are not significantly different during the forming phase and the axial feed phase, as the workpiece rotation continues because of the auxiliary rotary drive even after the tools get out of contact. Angular velocity keeps nearly constant, only in the moment of the initial die - workpiece contact a short change of acceleration is to be expected, as a small difference in circumferential velocity can be observed. At the moment when the tools get out of contact, the blocked axial feed and the angular motion are resumed and at the same time, so that the sensor moves along a helical path around the circumference of the cylindrical workpiece. Thus, an existing tooth profile will not be sampled on a circular path, but on a helical path. The geometrical information contained in the distance sensor's signal will relate one tooth after the other with an axial offset of the axial feed. Yet this fact cannot be detected in the sensor signal curve timing, as the rotational speed will be the same as during the forming cycle where no axial feed is superimposed.

The workpiece being held between two tips has certain concentricity deviation, which is composed of eccentricity plus roundness deviation, which both will be contained in any radial measurement. Roundness deviation of a cylindrical part can easily be measured before inserting a workpiece and by this measure it will become a priori known to the process, so that roundness deviation can be input to future process control software and could be subtracted from the measured concentricity value, yielding pure eccentricity. For the in-process measurement of eccentricity again a non-tactile distance sensor can be employed, which should be mounted in such a way, that it measures the radial distance to the surface of the workpiece during rotation (Fig. 10).

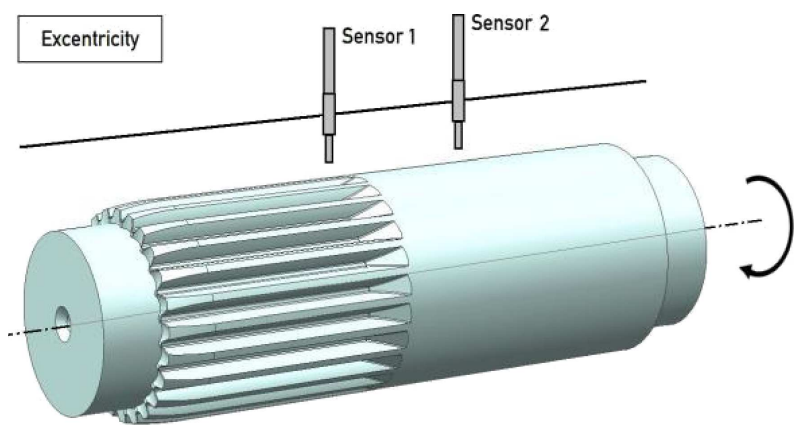

Fig. 10. Sensor placement for eccentricity determination.

The changes of this radial values versus the rotational angle of the workpiece, reduced by the roundness deviation of the blank results in the process quantity eccentricity. This measurement of eccentricity can be done during a forming cycle and during feed cycle as well. Fewer errors or interferences in 
the measurement signal can be expected, if the tools are not in contact, as this will eliminate most dynamic interferences caused by the forming process. Therefore, it is recommended to place the sensor in the zone outside the preforming and forming zone. It could also be an advantage to use two sensors, as shown, what would allow monitoring the formed zone of the workpiece and the zone outside the forming zone as well. Sensor 1 was placed in the forming zone to measure the distance to the surface of the workpiece. The sensor signal vs. time which is measured during workpiece rotation shows concentricity deviation, caused for example by a workpiece mounted eccentrically. Tactile measurement of the work pieces manufactured in the WPM forming process revealed that fluctuations of pitch and angular pitch have to be expected. Therefore, it is highly recommended to provide for an in-process detection and calculation of these parameters (Fig. 11). Pitch p is defined as the arc length at the perimeter of the pitch or reference circle from one toot to the next, or, the circumference of the pitch circle divided by the number of teeth.

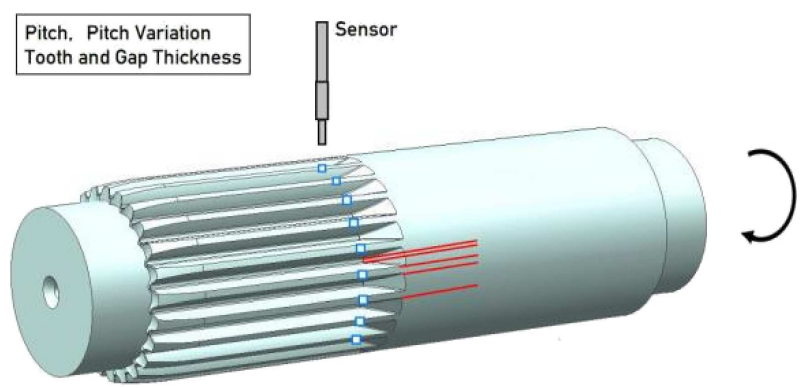

Fig. 11. Path of the sensor spot pitch variation/gap thickness.

The sensor signal of a suitable distance sensor will deliver a raw signal vs. time, which reflects duration and height of the teeth sampled. By comparison with an adjustable trigger levels, pulses can be generated at the rising and at the falling edge of the raw signal. This can be done in both ways, by hardware or by software. Figure 12 shows an example, where by use of two different trigger levels, so that pulses just above the base circle and pulse just below the tooth will be generated.

Distances shown on the figure are: tip pitch (Pa), tip thickness (Sa) and gap thickness (ef). For each of the two trigger levels, this results in two rising edge pulses (Sr, blue line) and two falling edge pulses (Sf, red line). Moreover, depending on individual requirements, also advanced methods of signal slope or edge detection could be applied [22].

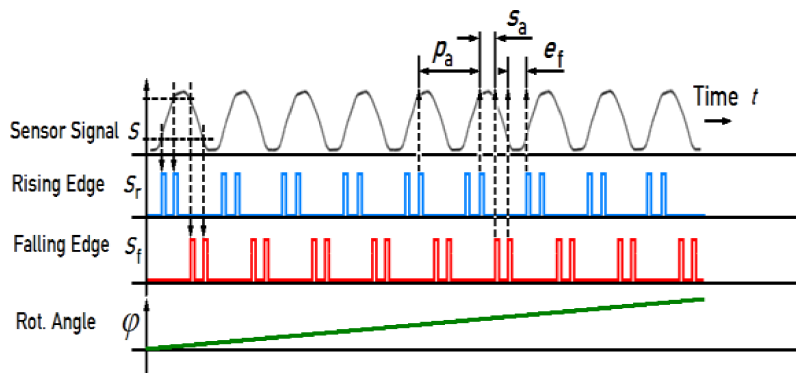

Fig. 12. Signal processing for geometrical peculiarities.

\section{Conclusions}

During measurements it was found out, that between the inlet at the beginning of the forming process, the central region and the end region of the metal forming results in considerable differences with respect to the height of head and the headshape. It was found that between two low tooth tips is always a high tooth. The high gear is always fully formed than the two adjacent teeth of low head height. This statement corresponds to a further realization, which affects the respective tooth shape. The resulting tooth shapes are dependent on the tooth height, so that typical tooth shape characteristics can be noted. With regard to the statement regarding the significance of the feed rate on the resulting geometry results the contour measurement, confirm the results of the measurements.

As the result of an analysis it was shown that a minimum of three resp. four quantities have to be measured. The acquisition of measuring data from two non-tactile distance sensors, one linear position sensor and one rotational angle sensor for measuring the angular position of the workpiece, as well as the process quantities derived from these quantities by in-process real time calculation was being discussed. The requirements regarding measuring range, accuracy and sample rate resulting from these considerations were determined resp. estimated. Which of the many sensors available on the market eventually could be selected according to these requirements, and the question if sensors having the required accuracy and sample rate do exist or have to developed, is beyond the scope of this work.

\section{References}

[1] Neugebauer R., Putz M., Hellfritzsch U., Improved process design and quality for gear manufacturing with flat and round rolling, CIRP Annals, 56/1, 307312, 2007. 
[2] Guenther A., Peters J., Numerical description of surfaces [in German], VDI-Berichte, 1880, 2001.

[3] Koenig W., Steffens K., Gear production by cold forming, CIRP Annals, 34, 481-483, 1985.

[4] Krapfenbauer H., Cold rolled precision toothed elements [in German], VDI-Berichte, 107-112, 1979.

[5] Weck M., Koenig W., Bartsch B., Production of involut-toothed gears by cold rolling, Repp. Conf. Proc., SME, 125-132, 1981.

[6] Eichner K.W., Dimension analytical considerations regarding cylinders [in German], PhD Thesis, THDarmstadt, Institut fuer Umformtechnik, 1981.

[7] Marciniak Z., Kopacz Z., New rotary metalworking processes developed in Poland, II International Conference on Rotary Metalworking Processes, 1982.

[8] Schmoeckel D., Hauk P., Development of a combined press-roll strategy to manufacture toothed forms [in German], VDI-Z Integrierte Produktion, 138, 46-48, 1996.

[9] Sladek J., Accuracy of coordinate measurements [in Polish], Cracow University of Technology, 2012.

[10] Goch G., Guenther A., Future Gear Metrology, VDIBerichte, 1665, 751-768, 2003.

[11] Budzik G. (ed.): Determination of momentary contact trace of gears using rapid prototyping [in Polish], Rzeszow University of Technology Press, Rzeszow, 2011.

[12] Lindner I., Eichner T., Sladek J., Wieczorowski M., In-Process quality control in metal forming of splined machine elements, 11th International Symposium on Measurement and Quality Control, Krakow - Kielce, 2013, ID 194.
[13] Fraden J., AIP Handbook of Modern Sensors: Physics, Designs and Application, AIP American Institute of Physics Press, New York, 1993.

[14] Dobosz M., Laser diode distance measuring interferometer - metrological properties, Metrology and Measurement Systems, XIX/3, 553-564, 2012.

[15] Kleeman L., Kuc R., An Optimal Sonar Array for Target Localization and Classification, IEEE International Conference on Robotics and Automation, San Diego, 3130-3135, 1994.

[16] Pedrick M., Tittmann B.R., Ultrasonic micrometer position indicator with temperature compensation, Ultrasonics Symposium IEEE, 1199-1202, 2004.

[17] Mathia T.G., Pawlus P., Wieczorowski M., Recent trends in surface metrology, Wear, 271, 3-4, 494508, 2011.

[18] Wieczorowski M., Mathia T., Carras S., Smierzchalski D., Surface topography inspection in multi-sensor approach, MOTSP 2014, Bol, Croatia, 2014, ID 147.

[19] Goch G., Gear Metrology, Annals of the CIRP, 50, 659-695, 2001.

[20] Brosed F.J., Aguilar J.J., Guillomia D., Santolaria J., 3D Geometrical Inspection of Complex Geometry Parts Using a Novel Laser Triangulation Sensor and a Robot, Sensors, 90-110, 2011.

[21] Gan Z., Tang Q., Visual Sensing and its application - Integration of Laser Sensors to Industrial Robots, Advanced Topics in Science and Technology in China, Springer \& Zhejiang University Press, 2011.

[22] Eichner T., Finding of geometrical parameters as a base of in-process metrology system in WPM gear forming, PhD Thesis, Cracow University of Technology, 2013. 